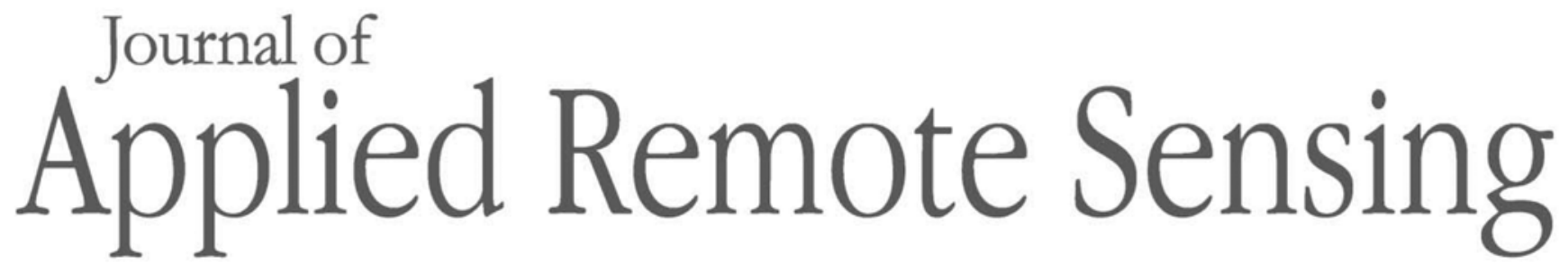

RemoteSensing.SPIEDigitalLibrary.org

\title{
Errata: Review of recent evaluation of satellite estimates sea surface salinity in the tropical Indian Ocean
}

\author{
Imranali M. Momin \\ Ashis K. Mitra \\ Debasis K. Mahapatra \\ Ekkattil N. Ragagopal
}




\title{
Errata: Review of recent evaluation of satellite estimates sea surface salinity in the tropical Indian Ocean
}

\author{
Imranali M. Momin, Ashis K. Mitra, Debasis K. Mahapatra, and \\ Ekkattil N. Ragagopal \\ National Centre for Medium Range Weather Forecasting (NCMRWF), \\ Earth System Science Organization (ESSO), Ministry of Earth Sciences (MoES) A-50, \\ Institutional Area, Sector-62, Noida 201309, India
}

[DOI: 10.1117/1.JRS.10.049901]

This paper [J. Appl. Remote Sens. 10(4), 046008 (2016)] was originally published with a grammatical error in the title. The title first appeared as "Review of recent evaluation of satellite estimates sea surface salinity in the tropical Indian Ocean." It has been changed to "Review of recent evaluation of sea surface salinity with satellite estimates in the tropical Indian Ocean." All online versions of the article were corrected on 21 November 2016.

(C) 2016 Society of Photo-Optical Instrumentation Engineers (SPIE) 